\title{
Vacuum Preparation, Optimization of Cylinder Length and Postoperative Daily Inflation Reduces Complaints of Shortened Penile Length Following Implantation of Inflatable Penile Prosthesis
}

\author{
Tom Sellers ${ }^{1}$, Martin Dineen ${ }^{1}$, Emad A. Salem ${ }^{2 \dagger}$, Steven K. Wilson ${ }^{3}$ \\ ${ }^{1}$ Daytona Beach, USA \\ ${ }^{2}$ Zagazig University, Zagazig, Egypt \\ ${ }^{3}$ Indio, CA, USA

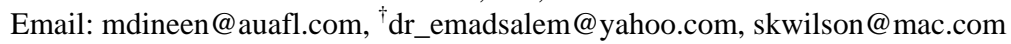

Received November 4, 2012; revised December 8, 2012; accepted December 16, 2012

\begin{abstract}
Introduction: The inflatable penile prosthesis (IPP) has been used to treat erectile dysfunction for 40 years. Loss of penile length following IPP remains the single biggest patient complaint. We describe a preoperative and postoperative patient preparation protocol to assist in setting realistic patient expectations and decreasing the complaint of reduced penile length. Materials \& Methods: 750 Patients are instructed to use a vacuum erection device for 10 minutes each day for up to 2 months prior to IPP implant. After two months, maximization of cylinder length is accomplished regardless of IPP manufacturer. Cylinders are left partially inflated in the post-operative period and daily inflation for 3 months immediately upon patient tolerance. The average implanted cylinder length has increased dramatically with the preoperative vacuum usage when compared to the authors' previous implantations and when compared to the national average of implanted cylinders obtained from one manufacturer. Results: Preoperative use of the vacuum device has allowed maximization of cylinder length. After the vacuum program, patients tend to experience less pain following implantation allowing earlier device instruction cycling and use. The average implanted cylinder length continued to increase annually for the first 5 years as the protocol evolved and seems to have remained stable for the last five years. Conclusions: Preoperative vacuum usage and postoperative capsule management has nearly eliminated patient complaints of reduced penile length. We believe this to be the result of larger size cylinders being implanted when compared to our previous implantations absent of the patient participation protocol.
\end{abstract}

Keywords: Inflatable Penile Prosthesis; Vacuum Device; Satisfaction

\section{Introduction}

The inflatable penile prosthesis (IPP) has been used to treat erectile dysfunction for 40 years. More than 250, 000 devices have been implanted and the device remains a durable solution for end organ failure. Despite availability of more conservative therapies such as pills, injections and vacuum devices, many physicians and patients turn to prosthesis implantation as the preferred therapy for severe erectile dysfunction. Penile implants are considered less often than more conservative therapies but when utilized, result in better patient satisfaction [1]. Current inflatable penile implants have been shown to require revision less often than most all other medical devices implanted in humans [2].

\footnotetext{
*Funds: There was no funding for this study.

\#TS: Coloplast and AMS speaker. MD: Coloplast and AMS speaker. ES None. SKW: Coloplast consultant.

${ }^{\dagger}$ Corresponding author.
}

Historically, device infection occurred in $4 \%$ of first time implantations, $8 \%$ of implants in diabetics and 10\% in revisions cases [3]. The use of antibiotic coatings has reduced infections approximately 50\% [4]. Penile shortening following successful prosthesis implantation when compared to the patient's memory of a natural erection remains the most common patient complaint [5]. Erect penile length that is not as long as prior to surgery is thought to be a result of capsule development around the cylinder that restricts the normal circumferential and lengthwise expansion of the tunica albuginea that occurs in a natural erection [6].

This study is a retrospective observational analysis of implant experience over the past ten years. We developed a pre and post-operative protocol of patient preparation that seems to markedly decrease the complaint of reduced penile length. This protocol facilitates the intraoperative experience for the surgeon by preparing the 
penis for acceptance of longer cylinders.

\section{Methods}

After disclosure of more conservative therapies and the subsequent decision by the patient to undergo surgery, the protocol is instituted. Patients are told the protocol is designed to diminish post-operative reduction in penile length. We emphasize that patient commitment to this training program of up to 5 months may be required (as many as two preoperatively and three postoperatively) to accomplish this goal. We are careful not to encourage unrealistic expectations of improved penile length when compared to their preoperative penile size. We stress the program is only meant to eliminate the common complaint of reduced penile length following IPP, not to provide penile enlargement. More than 750 patients have participated in the preparation protocol over the past 10 years.

After deciding to participate in the protocol, patients are instructed to use a vacuum erection device for $10 \mathrm{mi}$ nutes at least once daily for up to 2 months prior to surgery. If a patient has Peyronie's disease, shortening and fibrosis of the tunica is considered severe and he is instructed to use the device at least twice each day.

The penis is placed in the vacuum cylinder and the device pumped to the point where the patient feels a slight burning. The constriction ring is not applied and the penis experiences the vacuum for at least at least 10 minutes. Patient compliance is critical to the success of the preparation protocol. We encourage this by asking the patient to mark his lengthening progress with a permanent marker on the vacuum tube once each week (Figure 1).

To ensure a proper seal between the skin and the cylinder, the patient is instructed to shave around the base of the penis and to apply liberal amounts of lubricant on the penis and in the base of the vacuum cylinder. No bands or constriction rings are used. Patients are instructed to pause 3 - 5 seconds between pumps to achieve maximum benefit. The vacuum level is increased until the patient feels a slight burning sensation in the penis. The negative pressure is maintained for at least 10 minutes. A permanent marker is used to place a mark on the cylinder the first time the use of the vacuum device results in a penile erection. This allows patient and physician to quantify and monitor the patient's progress. The patient is asked to subsequently mark the vacuum cylinder weekly.

Not all patients wish to participate in the vacuum protocol. Some patients are eager to proceed with IPP implantation without delay. Others do not want to purchase the vacuum device. We attempt to convince these patients to participate in a truncated version of the program for only two weeks prior to surgery. We believe a period as short as two weeks will allow implantation of longer cylinders than would be possible without vacuum therapy,

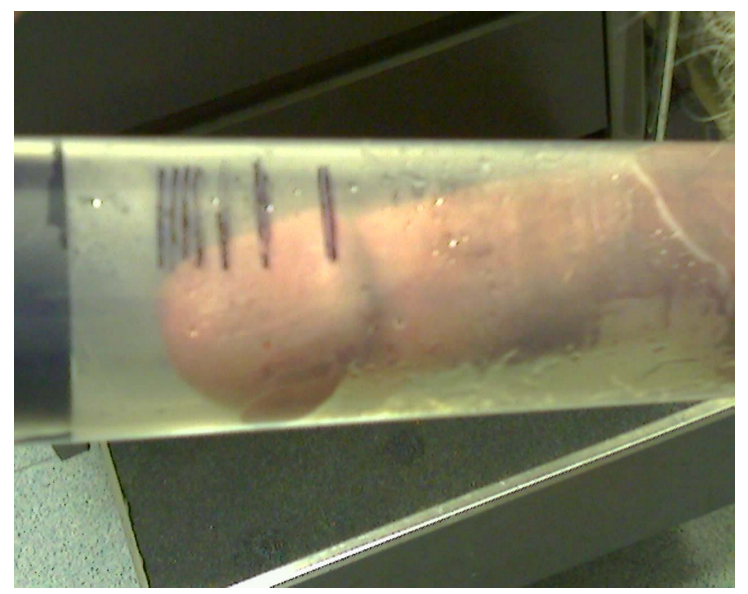

Figure 1. Photograph of patient marks on vacuum erection device. Note that weekly length gain is greatest during the first two weeks. Smaller gains continue up to eight weeks.

particularly in patients with recent onset of impotence e.g. post radical prostatectomy. Commonly, the largest expansion of penile length occurs in the first two weeks (Figure 1).

For the past several years we have also placed patients on the vacuum portion of the protocol who present for revision for mechanical failure prior to replacing their prosthesis. These patients were placed on the vacuum protocol for at least two weeks while awaiting revision surgery.

After the preoperative preparation protocol is completed, the patient is taken to the surgical suite. At the surgical table measurement of intracorporal distances are taken proximally and distally following dilatation to 12 $\mathrm{mm}$. During the distal measurement the penis is placed "on stretch" by vigorously pushing the measuring stick against the glans. We believe this maneuver "optimizes" our measurement. Our endpoint is to have no wrinkles in the cylinder when inflated. With this method of sizing cylinder length, some wrinkles are inevitably present in flaccidity.

Most of our patients are implanted on an outpatient basis. Following anesthesia recovery, the patient is discharged with his penis $50 \%$ erect, wrapped in a compression dressing, with a closed suction drain, and a Foley catheter. The Foley catheter is removed the following morning. After 48 hours, the patient returns to the office for bandage and drain removal. We usually deflate the IPP to $25 \%$, i.e. cylinders with no "wrinkling" along the shaft. This partial inflation is maintained for 9 - 12 days. The patient is asked to wear his penis up (cephalad) in brief type underwear to encourage capsule formation of the penis as in a natural erection.

Most patients are taught use of the device at $9-14$ days. They are instructed to daily inflate the device as much as possible for a period of up to one hour. Initially, many patients will only inflate for 7 - 10 minutes due to 
discomfort but the theory is that as much inflation as possible for as long as possible will influence the cylinder capsule to form around the inflated cylinder rather than the deflated cylinder. Postoperative inflation is emphasized strongly if a lengthening cylinder is implanted. Capsule development is thought to be complete at three months following surgery and the daily inflation can cease.

We compared patients undergoing our protocol with more than 235 patients in 2002 who were implanted before the institution of the vacuum preparation. We also obtained information from the two manufacturers of "average" cylinder size implanted across the United States in the years 2002-2007 and compared to our data.

\section{Results}

The average cylinder length has increased by more than $3.5 \mathrm{~cm}(18.4 \mathrm{~cm}$ vs $22.03 \mathrm{~cm})$ in our practice since institution of the patient participation protocol (Figure 2). We also exceeded the national "average" implant length of $18 \mathrm{~cm}$ by approximately $4 \mathrm{~cm}$. Notably, better increases in length were noted when we began "optimization" of cylinder size in 2006 and post operative inflation for an hour a day in 2007. Results of longer cylinder utilization also occurred in the revision patients. The revision surgery base showed increased cylinder length after undergoing vacuum treatment as reflected by their marking the vacuum tube. After vacuum therapy, at surgery, these patients had longer corporal measurements than during the original implantation and received longer cylinders (Figure 3).

\section{Discussion}

Use of the vacuum protocol has the potential to result in several benefits, both for the surgeon and the patient. The following subjective observations were noted when patients consistently followed vacuum protocol and the post-operative capsule manipulation as instructed.

- Increased ease of corpus cavernosum dilation

- Accommodation of larger cylinders

- Decreased postoperative pain

- Earlier postoperative cylinder inflation by the patient

- Realistic postoperative patient expectations

- Improved patient satisfaction with postoperative length It is our theory that daily use of the vacuum device prior to IPP reconditions the penis by drawing blood into the cavernosa and "exercising" the tunica. Recurrent exposure of the penis to vacuum therapy results in repetitive cavernosal engorgement that seems to make distal corporal cavernosal dilatation easier and allows the penis to accommodate a longer cylinder at the time of surgery. Vacuum prepared patients' cylinder length typically is up to $3.5 \mathrm{~cm}$ longer $(18.4 \mathrm{~cm}$ vs $22.03 \mathrm{~cm})$ when compared to patients not participating in vacuum therapy. Coloplast

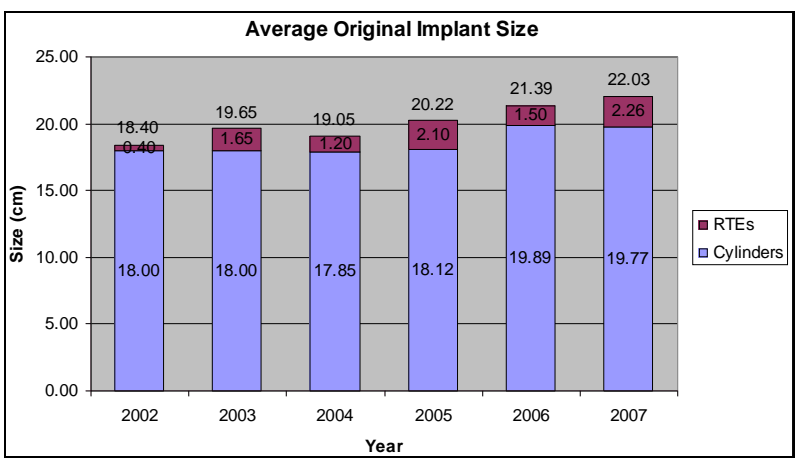

Figure 2. Comparison of non-protocol year 2002 with the next 5 years of protocol. 2006 marked beginning of "optimization of cylinder size and 2007 marked onset of daily post operative inflation.

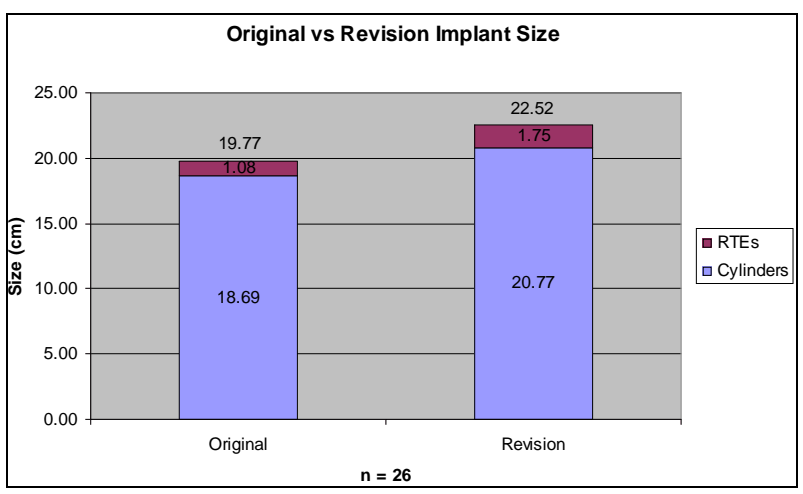

Figure 3. After 2 week vacuum preparation revision for mechanical failure, patients received longer cylinders.

one of the two American manufacturers of IPP devices indicate that the national "average" cylinder length implanted in the United States increased in 2002-2007 from $18.1 \mathrm{~cm}$ to $18.7 \mathrm{~cm}$. Today in our practice we average 22 $\mathrm{cm}$. amongst the patients employing the vacuum protocol.

At the surgical implantation, inflation of the cylinders by the surgeon seems easier when compared to patients who refused the protocol. Many of the patients implanted with an IPP have not experienced an erection for a number of months or even years and initial inflation in patients without the protocol requires considerable pressure. The authors have noted subjectively that the device "feels" easier to inflate in the vacuum participants. We believe postoperative care of the patient is also enhanced by regular preoperative use of the vacuum device. Patients are shown how to inflate/deflate the IPP shortly after surgery, typically on postoperative day 9 - 14. Vacuum prepared patients appear to experience less postoperative pain and therefore tend to have less difficulty cycling the pump at this early stage.

Admittedly, the previous paragraphs describe subjective opinions on the part of the authors. But these feelings have been reinforced over our ten years of experi- 
ence with this protocol because we still have a number of patients every year who refuse to follow the protocol and in effect serve as a "control" group. In this "control group" one of the authors (MKD) believes he can tell which patients have been faithful to the protocol and which has not by the ease of dilatation intra-operatively, ease of cylinder insertion, pace of recovery, perception of pain and ease of initial inflation.

Patients using the vacuum protocol seem satisfied with their postoperative penile length regardless of the type of cylinder implanted. We have used all the inflatable cylinders available from both manufacturers (AMS CX, AMS LGX, Mentor Alpha 1, Coloplast Titan) with vacuum preparation and no cylinder had better outcomes than the others. Early in our experience, we used a preponderance of lengthening cylinders (AMS LGX) believing it to theoretically be the ideal cylinder for this protocol. In recent years, however, we have no preference since the LGX failed to show superiority in patient satisfaction and can become a liability ("s" shape deformity) if the patient was not compliant with the post operative daily inflation (Figure 4).

Montague popularized a method of determining cylinder length in the 80's and 90's [7]. The distal measurement was taken from the top of the corporotomy and the proximal measurement marked from the bottom of the corporotomy. This in effect, downsized the length of the cylinder to be implanted by $2 \mathrm{~cm}$. Others in the urologic community believed cylinder length should be estimated by taking these measurements from the same point on the corporotomy, in effect, adding those $2 \mathrm{~cm}$ back [8].

We utilize the same point on the corporotomy but do so while stretching the penis, allowing maximization of cylinder length. We believe the tunica have been made more compliant by the vacuum therapy facilitating the stretching. It should be noted that we do not suggest we are over sizing the cylinders. When we size we are searching for a cylinder and Rear Tip Extender (RTE) combination that is well seated under the glans and provides a straight erection, with no "wrinkling" of the inflated cylinders at the time of implantation. Some wrinkling of the cylinder in flaccidity is witnessed in most cases and accepted.

Early in our observations we catalogued the various cylinder types manufactured by the two US companies to see if there was a difference in outcomes between the various cylinder models. AMS manufactures the LGX cylinder, which increases in girth to $18 \mathrm{~mm}$ and lengthens up to $20 \%$. Coloplast Titan is a cylinder that is unrestricted in girth and length expansion. Only the tunica limits Coloplast cylinders expansion and length. We wondered if the lengthening cylinder or the unrestricted expansion cylinder would have different outcomes after our vacuum protocol. Satisfaction with outcome seemed

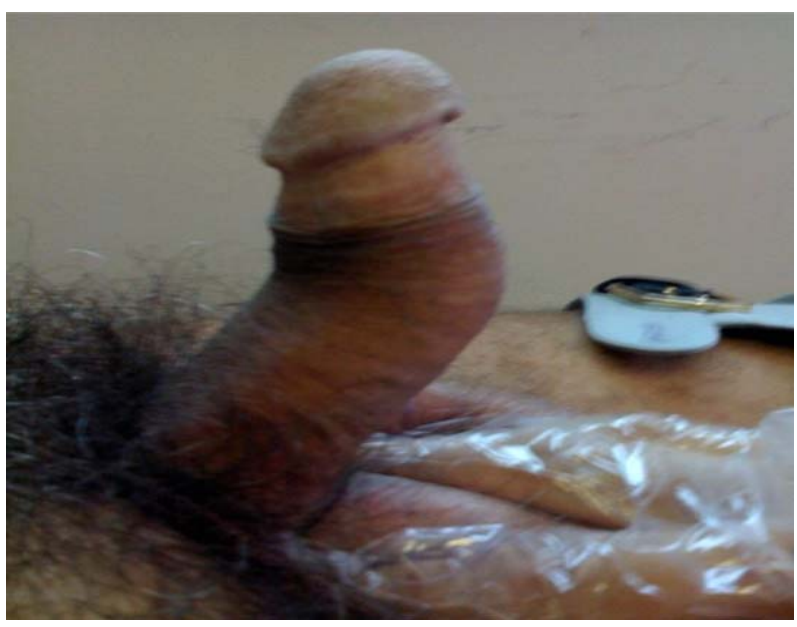

Figure 4. "s” shaped deformity of LGX cylinder.

similar with the different cylinder types and our cylinder choice currently is on a random basis except we avoid usage of the lengthening cylinder in cases of Peyronie's Disease [8].

Our results show patients receiving a short period of vacuum therapy prior to revision for mechanical failure demonstrate longer corporal measurements than when they were originally implanted. We recognize that the original implant has played some role as a "soft tissue expander". Wilson et al found constant pressure of a self contained inflatable enlarged the corporal measurements approximately $2 \mathrm{~cm}$ [9]. In this study, removal of a true 3-piece implant did not demonstrate increased corporal measurement at the time of revision. Nevertheless, most large volume implanters believe that, in most cases, a revision will accept longer cylinders than were implanted originally without vacuum preparation [10] and it seems questionable whether 2 weeks of vacuum application could influence the tough fibrotic capsule formed around the cylinders.

It is unfortunate that the vacuum protocol evolved over the past ten years and there was no effort to form a database, keep tract of cylinder type or quantitate satisfaction with a validated instrument. The hard data documenting increase of average cylinder size in our practice came from the manufacturer's Patient Information Form repository. Despite these considerable drawbacks, the authors are motivated to publish this article because we feel passionately that the protocol is a paradigm changing answer to improve patient outcomes.

\section{Conclusion}

Preoperative preparation of the penis with vacuum, optimization of cylinder length and postoperative daily inflation has decreased the patient complaint of reduced penile length following IPP surgery. We believe this improved patient satisfaction to be the result of preparing 
the tunica albuginea to accept larger cylinder length. Postoperative capsule manipulation by daily inflation for 3 months sustains the improvement. Patient satisfaction with penile size after using this protocol has been uniform regardless of the type of inflatable cylinder chosen.

\section{REFERENCES}

[1] A. Rajpurkar and C. Dhabuwala, "Comparison of Satisfaction Rates and Erectile Function in Patients Treated with Sildenafil, Intercavernosus Prostaglandin E, and Penile Implant Surgery for Erectile Dysfunction in Urology Practice," Journal of Urology, Vol. 170, 2003, pp. 159165. doi:10.1097/01.ju.0000072524.82345.6d

[2] S. K. Wilson, J. R. Delk, E. Salem and M. A. Cleves, "Long-Term Survival of Inflatable Penile Prosthesis: Single Surgical Group Experience with 2384 First-Time Implants Spanning Two Decades,” The Journal of Sexual Medicine, Vol. 4, No. 4, 2007, pp. 1074-1079. doi:10.1111/j.1743-6109.2007.00540.x

[3] S. K. Wilson and J. R. Delk, "Inflatable Penile Implant Infection: Predisposing Factors and Treatment Suggestions,” Journal of Urology, Vol. 153, 1995, pp. 659-663. doi:10.1016/S0022-5347(01)67678-X

[4] S. K. Wilson, J. Zumbe, G. D. Henry, et al., "Infection Reduction Using Antibiotic Coated Inflatable Penile Prosthesis,” Journal of Urology, Vol. 70, No. 2, 2007, pp. 337-340. doi:10.1016/j.urology.2007.03.058

[5] F. Montorsi, P. Rigatti, G. Carmignani, B. Campo, G. Ordesi, P. Silvestre, B. Giamusso, G. Morgia and A. Graziottin, "AMS Three-Piece Inflatable Implants for Erectile Dysfunction: A Long-Term Multi-Institutional Study in 200 Consecutive Patients," European Urology, Vol. 37,

\section{Key of Definitions for Abbreviations}

IPP: Inflatable penile prosthesis

RTE: Rear tip extender
No. 1, 2000, pp. 50-55. doi:10.1159/000020099

[6] F. Borges, L. Hakim and C. Kline, "Surgical Technique to Maintain Penile Length after Insertion of an Inflatable Penile Prosthesis,” The Journal of Sexual Medicine, Vol. 3, No. 3, 2006, pp. 550-553. doi:10.1111/j.1743-6109.2006.00232.x

[7] D. K. Montague, "Ultrex Cylinders: Problems with Uncontrolled Lengthening (The S-Shaped Deformity)," Journal of Urology, Vol. 155, No. 1, 1996, pp. 138-140.

[8] J. J. Mulcahy, "Prevention and Correction of Penile Implant Problems,” AUA Update Series, Lesson 27, 1994, pp. 214-218.

[9] S. K. Wilson, M. A. Cleves and J. R. Delk II, "LongTerm Follow-Up of a Treatment for Peyronie's Disease: Modeling the Penis over an Inflatable Penile Prosthesis," Journal of Urology, Vol. 165, 2001, pp. 825-831. doi:10.1016/S0022-5347(05)66537-8

[10] S. K. Wilson, M. A. Cleves and J. R. Delk, "Long-Term Results with Hydroflex and Dynaflex Penile Prostheses: Device Survival Comparison to Multi-component Inflatables,” Journal of Urology, Vol. 155, 1996, pp. 16211625. doi:10.1016/S0022-5347(01)66145-7

[11] S. K. Wilson, J. R. Delk II, J. J. Mulcahy, M. Cleves and E. A. Salem, "Upsizing of Inflatable Penile Implant Cylinders in Patients with Corporal Fibrosis," The Journal of Sexual Medicine, Vol. 3, No. 4, 2006, pp. 736-742. doi:10.1111/j.1743-6109.2006.00263.x 